\title{
Coronavirus Disease-19 Testing Strategies for Patients and Health Care Workers to Improve Workplace Safety
}

Sanghamitra Mohanty, MD, MS ${ }^{a}$, Uriel Garcia, BS ${ }^{b}$, Bryan MacDonald, MD Angel Mayedo, $\mathrm{MD}^{\mathrm{a}}$, Domenico G. Della Rocca, $\mathrm{MD}, \mathrm{PhD}^{\mathrm{a}}$, Carola Gianni, MDa ${ }^{a}$ Patrick Udenyi, BS`, William Zagrodzky, BS ${ }^{a}$, SaiShishir Shetty, DPharm, MHI ${ }^{a}$, Andrea Natale, MD ${ }^{a, d, e, *}$

\section{KEYWORDS}

- COVID-19 • SARS-CoV-2 • Transmission • Viral shedding • Antibody assay • Viral antigen test - Screening

\section{KEY POINTS}

- Universal testing at the workplace and subsequent home-isolation of identified cases successfully lowers the risk of horizontal transmission and creates a safe working environment

- In health care settings, close proximity while working and prolonged working hours make health care workers vulnerable to contracting the infection

- Presymptomatic and asymptomatic cases make it challenging to identify COVID-19 cases in the conventional symptom-based screening, reinforcing the usefulness of viral antigen, antibody, and RNA-based tests

Background: COVID-19 has been declared as a global health emergency with hundreds of millions being affected and many succumbed to this infectious disease since its first detection in China in December 2019. ${ }^{1}$ Rapid emergence of mutant strains, high infection rate, and infection-mortality ratio coupled with limited knowledge about the severe acute respiratory syndrome coronavirus 2 (SARS-CoV-2) have made the management of this pandemic extremely challenging. ${ }^{2}$ Subsequently, public health measures such as social distancing, universal use of facial masks, and restrictions at workplaces with shut-downs and lock-downs of many businesses have been imposed to limit the spread. However, the pandemic is far from being under control because of a continued surge of new variants and as a substantial share of the global population is still not immunized either due to their personal preferences or unavailability of the vaccine. Fearful patients suffering from other serious morbidities, with the intention of avoiding this highly contagious disease, reporting later in the disease progression resulting in poor outcome is another important collateral adversity of COVID-19. ${ }^{2}$ Under this circumstance, with very little information to predict the course of this pandemic that has not yet shown any signs of remission, it is

\footnotetext{
a Texas Cardiac Arrhythmia Institute, St. David's Medical Center, Austin, TX, USA; ${ }^{b}$ Universidad Anahuac, Mexico City, Mexico; ${ }^{c}$ University of California - Berkeley, Berkeley, CA, USA; ${ }^{d}$ Interventional Electrophysi-

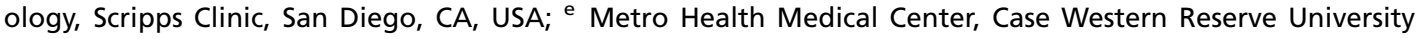
School of Medicine, Cleveland, OH, USA

* Corresponding author. 3000 North I-35, Suite 720, Austin, TX 78705.

E-mail address: dr.natale@gmail.com
} 


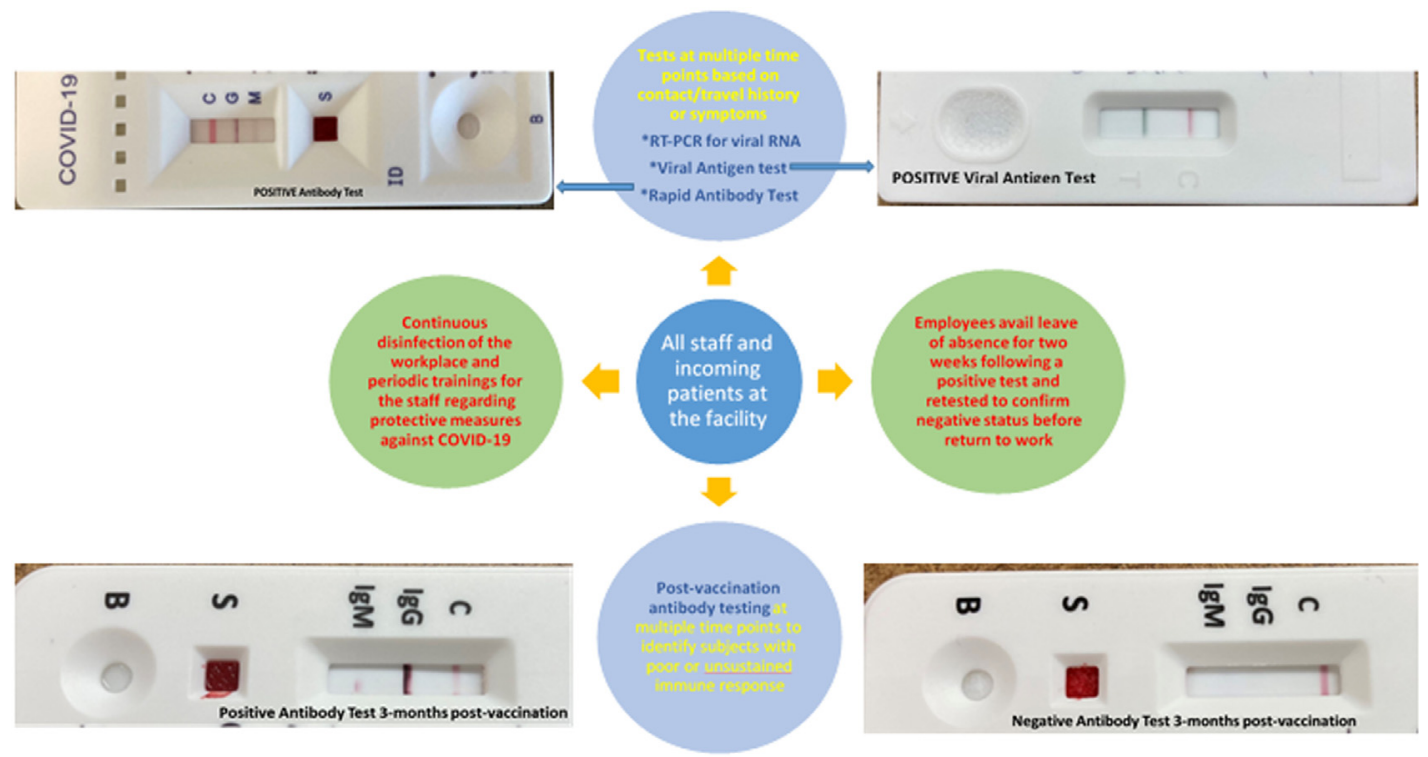

Fig. 1. Schematic showing strategies to create a COVID-19-free workplace.

highly crucial to find ways to create a safe environment for both patients and health care workers so that the health care institutions can continue providing the best care while protecting the health and wellbeing of all personnel involved and saving the community from the devastating toll of this pandemic on the economy and social fabric. This paper provides a brief overview of findings reported by different groups to mitigate workplace transmission of COVID-19.

Discussion: Viral shedding and transmission can happen in symptomatic as well as asymptomatic individuals infected with SARS-CoV-2 virus. ${ }^{3}$ Shedding of replication-competent SARS-CoV-2 is believed to occur for 2 to 3 weeks following symptom onset and often much beyond the end of symptoms. ${ }^{3}$ Moreover, presymptomatic and asymptomatic transmission can account for approximately $40 \%-45 \%$ of SARS-CoV-2 infections. ${ }^{3}$ The characteristics of SARS-CoV-2 such as higher transmissibility, longer incubation period, asymptomatic transmission, and prolonged viral shedding illustrate the potential role of the workplace in SARS-CoV-2 transmission. ${ }^{3}$ According to the Centers for Disease Control (CDC) guidelines, health care workers are at a very high risk for contracting this infection, which is not only while taking care of patients but most plausibly also by working closely with coworkers with unknown infection/contact status, for long hours. ${ }^{4}$ In the beginning of the pandemic, of the 138 patients treated at Wuhan Hospital in China, $40(29 \%)$ were health care workers. ${ }^{4}$ Two systematic reviews reported up to $11 \%$ of the global
COVID-19 cases to be health care workers. ${ }^{5}$ One prospective independent study documented health care workers in United Kingdom and United States to be at a significantly higher risk of contracting the infection than the general population. ${ }^{6}$ Another trial by Schneider and colleagues demonstrated multiple unprotected contacts between infected staff to be responsible for 4 outbreaks in their hospital. ${ }^{7}$

Because of the silent spread of this viral infection by asymptomatic and pre- and postsymptomatic persons, besides vaccination, social distancing and utilization of hygienic measures, universal testing, screening, and surveillance are highly critical in effectively identifying the source of infection and thus preventing horizontal transmission in the target community.

In a multi-center study, we evaluated the efficacy of widespread screening in creating a safe workplace in a consecutive series of 1670 asymptomatic subjects (no symptoms of COVID-19) including patients and their caregivers and staff in our electrophysiology (EP) units. ${ }^{2}$ Furthermore, we assessed the new infection rate in patients undergoing EP procedure, to see if identification and exclusion of positive cases facilitated the establishment of a risk-free operating environment. ${ }^{2}$ All received serologic testing using COVID-19 Rapid Antibody Assays (Premier Biotech Labs, 723 Kasota Avenue SE, Minneapolis, MN 55414 and Confirm Biosciences, San Diego, CA) and nasopharyngeal (NP) swab was tested for viral RNA using ID NOW test kit from Abbott Diagnostics Inc. (Scarborough, Maine). All test kits were cross- 
verified internally by testing them against known COVID-19 positive and negative patients. Staff testing positive for viral-RNA on day 0 were asked for 2-week of self-isolation and were retested on day 14 before they got back to work. In 2 hospitals, personnel involved in patient care including technicians, nurses, physicians, dieticians, janitorial staff were retested at 14 to 21 days using an NP swab test for viral-RNA. Patients undergoing EP procedures were followed up for 2 weeks after being discharged from the hospital. They were asked to measure daily temperatures and report any symptoms of COVID-19 if experienced during the following 2-week period.

In our series, 64 (64/1670) cases tested positive for viral RNA at baseline, of which 33 were health care workers. All 33 staff members testing positive on day 0 were asked to self-quarantine for 2 weeks and were retested for viral RNA on day $14 ; 32 / 33$ $(96.9 \%)$ were negative and were allowed to get back to work. One staff with positive viral-RNA on day 14 tested negative on day 21 and returned to work then. None of the patients that received EP procedures during the study period reported symptoms of COVID-19 infection within 2 weeks after being discharged from the hospital.

A total of 67 patients were retested because of potential exposure to COVID-19 cases; 6 of the 67 tested positive. In these 6 positive cases, contact tracing revealed the possible source of infection to be family members or friends outside of the workplace. None of the coworkers of these 6 staff tested positive at that time.

As the findings demonstrate, universal testing and screening of all incoming patients, their caregivers, and hospital staff helped in identifying and isolating positive cases, thereby creating a safe working environment within the confines of our EP units.

In another observational study conducted in Wuhan, China, health screening (nucleic acid test for SARS-CoV-2, antibody detection, and chest CT scan) for COVID-19 was conducted among all returning staff from March to May 2020 that identified asymptomatic infection in some. ${ }^{8}$ Close contacts of those positive cases were tracked, thereby curbing the risk of potential transmission at the workplace. ${ }^{8}$

Symptom screening before work entry is widely practiced in workplaces. However, high prevalence of asymptomatic illness attenuates the effectiveness of this strategy. ${ }^{9}$ Haigh and colleagues reported the benefits of nonpharmaceutical interventions along with testing campaigns via reverse transcription-polymerase chain reaction (RT-PCR) of nasal swabs in late July to early August 2020, in 586 employees at 3 sites. ${ }^{9}$ True positivity rates were consistent with community prevalence at the time; of all employees with positive tests, $99 \%$ were asymptomatic. ${ }^{9}$ In a metaanalysis of 97 studies including 230,398 health care workers, estimated prevalence of SARSCoV-2 infection was $11 \%$ and among the RTPCR positive cases, $40 \%$ did not show symptoms at the time of diagnosis. ${ }^{10}$

As a high percentage of health care workers are vaccinated at this point and the overall epidemic wave is receding in countries, prospective patients should feel reassured about the low risk of contracting the infection in a health care setting. However, the risk still remains for those that are poor responders to COVID-19 vaccination or those that are unvaccinated. In an ongoing trial conducted by our group, $\sim 85 \%$ of the staff were seen to have moderate to strong antibody (immunoglobulin $\mathrm{G}$ ) response at 6 months following the second dose of COVID-19 vaccine (unpublished data). Thus, the antibody testing has also helped in identifying individuals lacking sustained immune response that could be vulnerable to future infection and would plausibly need booster doses for further protection.

These data reinforce the importance of multitime point surveillance and screening of staff to create a COVID-19-free workplace in health care settings. ${ }^{11}$

Summary: Universal testing for COVID-19, at different time points as needed based on the contact history and/or symptoms would facilitate the prevention of horizontal transmission and creation of a safe workplace by identifying and isolating infectious cases. Given the continuing emergence of new viral strains with increased infectivity and severity and uncertainty of the efficacy of the available vaccines in providing protection against those new variants, the significance of widespread screening cannot be overemphasized while preparing workplaces worldwide for the safe return of employees and patients.

\section{CLINICS CARE POINTS}

To create a COVID-19-free workplace (Fig. 1).

- Employers should provide universal testing (RT-PCR for viral RNA, viral antigen, and antibody tests as deemed appropriate) facilities for all staff at multiple time points based on their travel/contact history and/or presenting symptoms

- Employees should consider taking leave of absence from work for 2 weeks after testing positive for viral antigen regardless of the 
symptom-status and repeat tests to confirm a negative result for COVID-19 before returning to work

- Vaccinations against COVID-19 should be highly recommended and antibody testing following vaccination should be performed at 3,6 , and 12 months to identify subjects with poor or unsustained immune response.

- Continuous disinfection of the workplace and periodic training for the staff regarding safety measures such as hand-washing, facial coverings, and timely reporting of their travel/contact history must diligently ensue

\section{DISCLOSURES}

Dr A. Natale is a consultant for Boston Scientific, Biosense Webster, St. Jude/Abbott Medical, Biotronik, Baylis, and Medtronic. Remaining authors have nothing relevant to disclose.

\section{REFERENCES}

1. Rueda-Garrido JC, Vicente-Herrero T, del Campo T, et al. Return to work guidelines for the COVID-19 pandemic. Occup Med 2020;70:300-5.

2. Mohanty S, Lakkireddy D, Trivedi C, et al. Creating a safe workplace by universal testing of SARS-CoV-2 infection in asymptomatic patients and healthcare workers in the electrophysiology units: a multi-center experience. J Interv Card Electrophysiol 2020;1-6. https://doi.org/10.1007/s10840-020-00886-9.

3. Plantes PJ, Fragala MS, Clarke C, et al. Model for mitigation of workplace transmission of COVID-19 through population-based testing and surveillance. Popul Health Manag 2021;24(S1):S16-25.

4. Rafeemanesh E, Ahmadi F, Memarzadeh M. A review of the strategies and studies on the prevention and control of the new Coronavirus in workplaces. Arch Bone Jt Surg 2020;8(Suppl1):242-6.
5. Sahu AK, Amrithanand VT, Mathew R, et al. COVID19 in health care workers - a systematic review and meta-analysis. Am J Emerg Med 2020 Sep;38(9): 1727-31.

6. Nguyen LH, Drew DA, Joshi AD, et al. Risk of COVID-19 among frontline healthcare workers and the general community: a prospective cohort study. medRxiv 2020. https://doi.org/10.1101/2020.04.29. 20084111.

7. Schneider S, Piening B, Nouri-Pasovsky PA, et al. SARS-Coronavirus-2 cases in healthcare workers may not regularly originate from patient care: lessons from a university hospital on the underestimated risk of healthcare worker to healthcare worker transmission. Antimicrob Resist Infect Control 2020;9(1):192. https://doi.org/10.1186/s13756020-00848-w.

8. Duan P, Deng ZQ, Pan ZY, et al. Safety considerations during return to work in the context of stable COVID-19 epidemic control: an analysis of health screening results of all returned staff from a hospital. Epidemiol Infect 2020;148:e214. https://doi.org/10. 1017/S0950268820002150.

9. Haigh KZ, Gandhi M. COVID-19 mitigation with appropriate safety measures in an Essential workplace: lessons for Opening work settings in the United States during COVID-19. Open Forum Infect Dis 2021;8(4):ofab086. https://doi.org/10.1093/ofid/ ofab086.

10. Gómez-Ochoa SA, Franco OH, Rojas LZ, et al. COVID-19 in health-care workers: a Living systematic review and meta-analysis of prevalence, risk Factors, Clinical characteristics, and outcomes. Am J Epidemiol 2021;190(1):161-75.

11. Treibel TA, Manisty C, Burton M, et al. COVID-19: PCR screening of asymptomatic health-care workers at London hospital. Lancet 2020; 395(10237):1608-10. 